\title{
Carbonylative annulation of unsaturated compounds using molybdenum hexacarbonyl: an efficient synthesis of $2(1 \mathrm{H})$ - quinolones
}

\author{
Farnaz Jafarpour, ${ }^{\mathrm{a} *}$ and Asieh Otaredi-Kashani ${ }^{\mathrm{a}, \mathrm{b}}$ \\ ${ }^{a}$ School of Chemistry, College of Science, University of Tehran, 14155-6455 Tehran, Iran \\ ${ }^{\mathrm{b}}$ Building and Housing Research Center, 13145-1696 Tehran, Iran \\ E-mail: jafarpur@khayam.ut.ac.ir
}

DOI: http://dx.doi.org/10.3998/ark.5550190.p008.531

\begin{abstract}
A ligand- and $\mathrm{CO}$ gas-free condition is developed in palladium-catalyzed three-component reaction of iodoanilines, unsaturated compounds and $\mathrm{Mo}(\mathrm{CO})_{6}$ as a solid carbon monoxide source. The approach allows for smooth construction of biologically interesting 3,4-disubstituted (dihydro)quinolin-2(1H)-ones in presence of catalytic amounts of palladium and avoids the problematic use of gaseous carbon monoxide.
\end{abstract}

Keywords: 2-Quinolone, palladium, carbonylation, $\mathrm{Mo}(\mathrm{CO})_{6}$, iodoaniline, internal alkyne, norbornene

\section{Introduction}

Palladium-catalyzed carbonylation reactions as a highly efficient and selective way for converting fine chemicals into a diverse range of products have found widespread use in organic synthetic applications. ${ }^{1-6}$ Introduction of a carbonyl functionality into organic molecules which is pioneered by Heck and co-workers ${ }^{7-8}$ and combination of the process with subsequent intramolecular cyclization reactions, have permitted an efficient access to various biologically interesting heterocycles. ${ }^{9-16}$

Although carbonylations of a wide spectrum of organometallic reagents have been performed using gaseous carbon monoxide but the use of highly toxic flammable gaseous carbon monoxide is often impractical as it associates with risky and troublesome handling and needs highly specialized equipments. According to limited synthetic applications of gaseous carbon monoxide, employing alternative $\mathrm{CO}$ sources including metal carbonyls generating carbon monoxide in situ during the reaction would be more appealing. Among them, $\mathrm{Mo}(\mathrm{CO})_{6}$ represents an ideal easily handled solid reagent for in situ release of carbon monoxide. In this regard, several groups have reported on various CO-gas free methods including microwave- 
assisted palladium-catalyzed $\mathrm{Mo}(\mathrm{CO})_{6}$-mediated carbonylative coupling reactions. ${ }^{17-32}$ Recently we also reported a facile route to diaryl ketones through palladium-catalyzed three-component cross-coupling of aryl and heteroaryl halides, $\mathrm{Mo}(\mathrm{CO})_{6}$ and boronic acids and offered a mild base/solvent combination for efficient microwave-free extrusion of carbon monoxide in the course of the reaction. ${ }^{33}$ Continuing our interest in carbonylation reactions, we further attempted to synthesize (dihydro)quinolones via carbonylative annulation reactions of readily available 2iodoanilines and unsaturated compounds. Quinolones are interesting structural motifs found in numerous biologically active natural products. Several synthetic quinolinone derivatives have been also found to possess various pharmacological activities. ${ }^{34-35}$ For instance compounds of type $\mathbf{1}$ and $\mathbf{2}$ are known to be selective Androgen receptor modulators and to have positive inotropic effects, respectively (Figure 1). ${ }^{36}$ Linomide 3, a synthetic immunomodulator, has been also investigated in treatment of various types of cancers and autoimmune disorders (Figure 1). ${ }^{37}$ Thus, general and versatile synthetic methods for construction of these interesting frameworks are of great interest in synthetic organic chemistry.

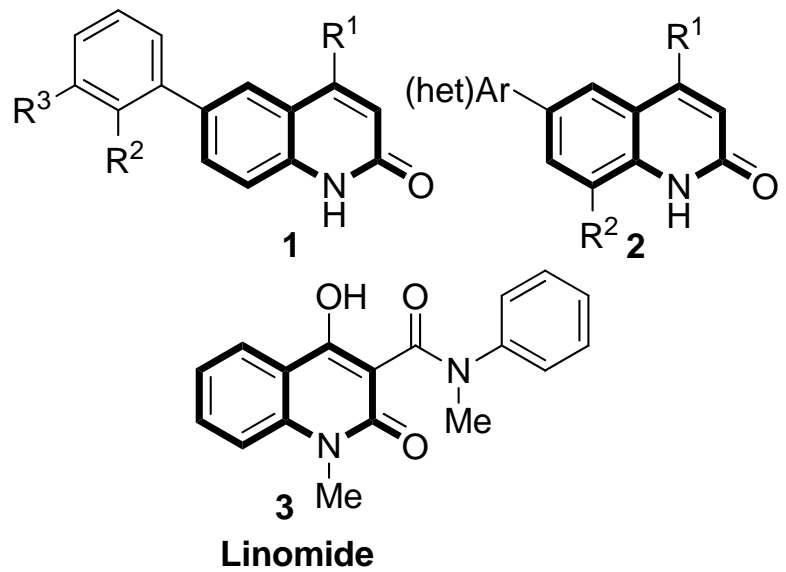

Figure 1. Biologically active quinolones.

Although various classic methodologies exist for construction of 2 -quinolinones, ${ }^{38-47}$ their utility in construction of 3,4-disubstituted quinolones are limited and they usually lead to monosubstituted scaffolds. On the other hand, only few examples of metal-catalyzed strategies which provide more powerful and practical route to heterocycles, are reported for construction of substituted quinolones. ${ }^{48-60}$ Some recent developments in metal-catalyzed construction of these privileged scaffolds include cycloaddition of o-cyanophenylbenzamides ${ }^{61-62}$ and $\mathrm{N}$ arylcarbamoyl chlorides ${ }^{63}$ with internal alkynes, ring closing metathesis (RCM) reaction of $\mathrm{N}$ phenylacrylamides, ${ }^{64}$ domino Heck/Buchwald-Hartwig reaction of o-bromocinnamamide and iodoarenes $^{65}$ and cyclization of 3,3-diarylacrylamides through intramolecular C-H amination ${ }^{66}$ which often require employing complicated starting materials. Larock et al. developed a palladium-catalyzed carbonylative annulation of alkynes with readily available anilines employing gaseous CO. ${ }^{67-68}$ Recently Xiao et al. further developed this strategy through a 
microwave- assisted $\mathrm{Pd}(\mathrm{OAc})_{2} /$ dppe catalyzed carbonylative annulation of terminal alkynes with iodoanilines using solid carbon monoxide source. ${ }^{69}$ The protocol however encountered some limitations where no reactivity was observed with internal alkynes or N-protected 2-iodoanilines and the approach typically led to only monosubstituted quinolones.

Prompted by the recent advances in $\mathrm{CO}$ gas-free carbonylative reactions and continuing our interest in the field, we attempted to construct 3,4-disubstituted quinolone scaffold through a palladium-catalyzed three-component reaction of iodoaniline, internal alkynes/alkenes and $\mathrm{Mo}(\mathrm{CO})_{6}$ as a solid carbon monoxide source. The protocol is experimentally simple, uses readily available starting materials and is a ligand and microwave-free choice for construction of 3,4disubstituted (dihydro)quinolin-2(1H)-ones. Furthermore, the annulation reactions proceed with insertion of unsaturated compounds into the arylpalladium bond in preference to insertion of carbon monoxide where no isomeric quinolones were observed.

\section{Results and Discussion}

Initially when iodoaniline 1a and norbornene $\mathbf{2 a}$ where reacted under the conditions applied for construction of diarylketones, only traces of the desired product 3a was obtained. Next optimization reactions with respect to catalyst, base and solvents were conducted (Table 1). When $\mathrm{PdCl}_{2}$ as the catalyst and DBU as an organic base in presence of an additive (TBAC) in THF were employed, a $30 \%$ of the desired annulated product 3a was obtained (entry 1). Upon optimization of the palladium catalyst and base, $\operatorname{Pd}(\mathrm{OAc})_{2}$ and py showed superior reactivity (entries 2-5). As screening reactions with respect to ligands including $\mathrm{PPh}_{3}, \mathrm{Pcy}_{3}, \mathrm{Pcy}_{3} . \mathrm{HBF}_{4}$, dppe and TFP, resulted in only comparable or lower yields of tricyclic product, ligand-free condition was established for later investigations (entries 6-10). Finally, between various solvents investigated, the carbonylation/cyclization reaction proceeded in higher yield in DMF (entries 11-15). The optimal conditions of o-iodoaniline, norbornene (5 equiv.), py (3 equiv.), $\mathrm{Pd}(\mathrm{OAc})_{2}(10 \mathrm{~mol} \%)$ and $\mathrm{Mo}(\mathrm{CO})_{6}\left(1.5\right.$ equiv.) in $\mathrm{DMF}$ at $160{ }^{\circ} \mathrm{C}$ for $12 \mathrm{~h}$, led to formation of hexahydrophenanthridinone $3 \mathbf{a}$ in $45 \%$ isolated yield (Table 2, entry 1). It is noteworthy that norbornene adds to the arylpalladium intermediate in a cis-exo manner followed by $\mathrm{CO}$ insertion and intramolecular amination. The slow CO liberation in the course of reaction also provides an efficient pressure of the gas to promote the carbonylative reaction. This protocol merges three Heck, carbonylation and amination reactions to construct two $\mathrm{C}-\mathrm{C}$ and one $\mathrm{C}-\mathrm{N}$ bonds in one pot. 
Table 1. Optimization of carbonylative annulation reaction ${ }^{\mathrm{a}}$

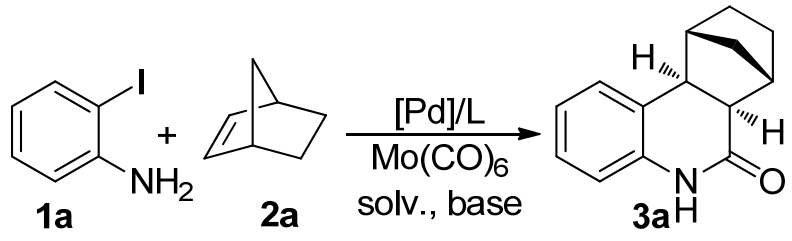

\begin{tabular}{cccccc}
\hline Entry & {$[\mathrm{Pd}]$} & Base & $\mathrm{L}$ & Solv. & Yield (\%) \\
\hline 1 & $\mathrm{PdCl}{ }_{2}$ & $\mathrm{DBU}$ & & $\mathrm{THF}$ & 30 \\
2 & $\mathrm{Pd}(\mathrm{OAc})_{2}$ & $\mathrm{DBU}$ & & $\mathrm{THF}$ & 33 \\
3 & $\mathrm{Pd}(\mathrm{OH})_{2} / \mathrm{C}$ & $\mathrm{DBU}$ & & $\mathrm{THF}$ & 15 \\
4 & $\mathrm{Pd}(\mathrm{dppe})_{2}$ & $\mathrm{DBU}$ & & $\mathrm{THF}$ & 25 \\
5 & $\mathrm{Pd}(\mathrm{OAc})_{2}$ & py & & $\mathrm{THF}$ & 36 \\
6 & $\mathrm{Pd}(\mathrm{OAc})_{2}$ & py & $\mathrm{PPh}_{3}$ & $\mathrm{THF}$ & 31 \\
7 & $\mathrm{Pd}(\mathrm{OAc})_{2}$ & py & $\mathrm{Pcy}_{3}$ & $\mathrm{THF}$ & 25 \\
8 & $\mathrm{Pd}(\mathrm{OAc})_{2}$ & py & $\mathrm{Pcy}_{3} . \mathrm{HBF}_{4}$ & $\mathrm{THF}$ & 28 \\
9 & $\mathrm{Pd}(\mathrm{OAc})_{2}$ & py & $\mathrm{dppe}_{2}$ & $\mathrm{THF}$ & 22 \\
10 & $\mathrm{Pd}(\mathrm{OAc})_{2}$ & py & $\mathrm{TFP}_{2}$ & $\mathrm{THF}$ & 12 \\
11 & $\mathrm{Pd}(\mathrm{OAc})_{2}$ & py & & $\mathrm{ACN}$ & 35 \\
12 & $\mathrm{Pd}(\mathrm{OAc})_{2}$ & py & & $\mathrm{DMA}$ & 35 \\
13 & $\mathrm{Pd}(\mathrm{OAc})_{2}$ & py & & DME & 33 \\
14 & $\mathrm{Pd}(\mathrm{OAc})_{2}$ & py & & Toluene:ACN & 41 \\
15 & $\mathrm{Pd}(\mathrm{OAc})_{2}$ & py & & DMF & $45^{\mathrm{b}}$ \\
\hline
\end{tabular}

${ }^{a}$ Reactions conditions: o-Iodoaniline $(0.2 \mathrm{mmol})$, norbornene $(1.0 \mathrm{mmol})$, Base $(0.6 \mathrm{mmol})$, $\mathrm{n}$ $\mathrm{Bu}_{4} \mathrm{NCl}(0.2 \mathrm{mmol})$, catalyst $(10 \mathrm{~mol} \%)$, ligand $(20 \mathrm{~mol} \%)$ and $\mathrm{Mo}(\mathrm{CO})_{6}(0.3 \mathrm{mmol})$, solvent $(2$ $\mathrm{mL})$ at $100{ }^{\circ} \mathrm{C}$ for $12 \mathrm{~h} .{ }^{\mathrm{b}} 160{ }^{\circ} \mathrm{C}$.

Next protection of the amino group of the aniline was explored. Gratifyingly, with an ethoxycarbonyl protecting group, tricyclic adduct 3a was obtained in almost quantitative yield (95\%, entry 2). Ethyl ester deprotection proceeded in the course of the reaction and completed during the workup. Encouraged by the results, we explored some other protecting groups. The carbonylative annulation reaction of norbornene with $\mathrm{N}$-tosyl-o-iodoaniline afforded the desired product in $80 \%$ yield (entry 3 ). Sulfonyl and acyl protecting groups also resulted in the annulated products in 85 and $87 \%$ yields, respectively (entries 4-5). Next internal alkynes were employed to investigate the scope and limitations of the approach in constructing 3,4-disubstituted quinolones. Although annulation reaction of dipropyl acetylene with unprotected iodoaniline afforded only $25 \%$ of the desired quinolone $3 \mathbf{b}$, but protected anilines resulted in the same product in high to excellent yields exceeding $85 \%$ (entries 6-9). 
Table 2. Scope of annulation reactions of N-substituted o-iodoanilines

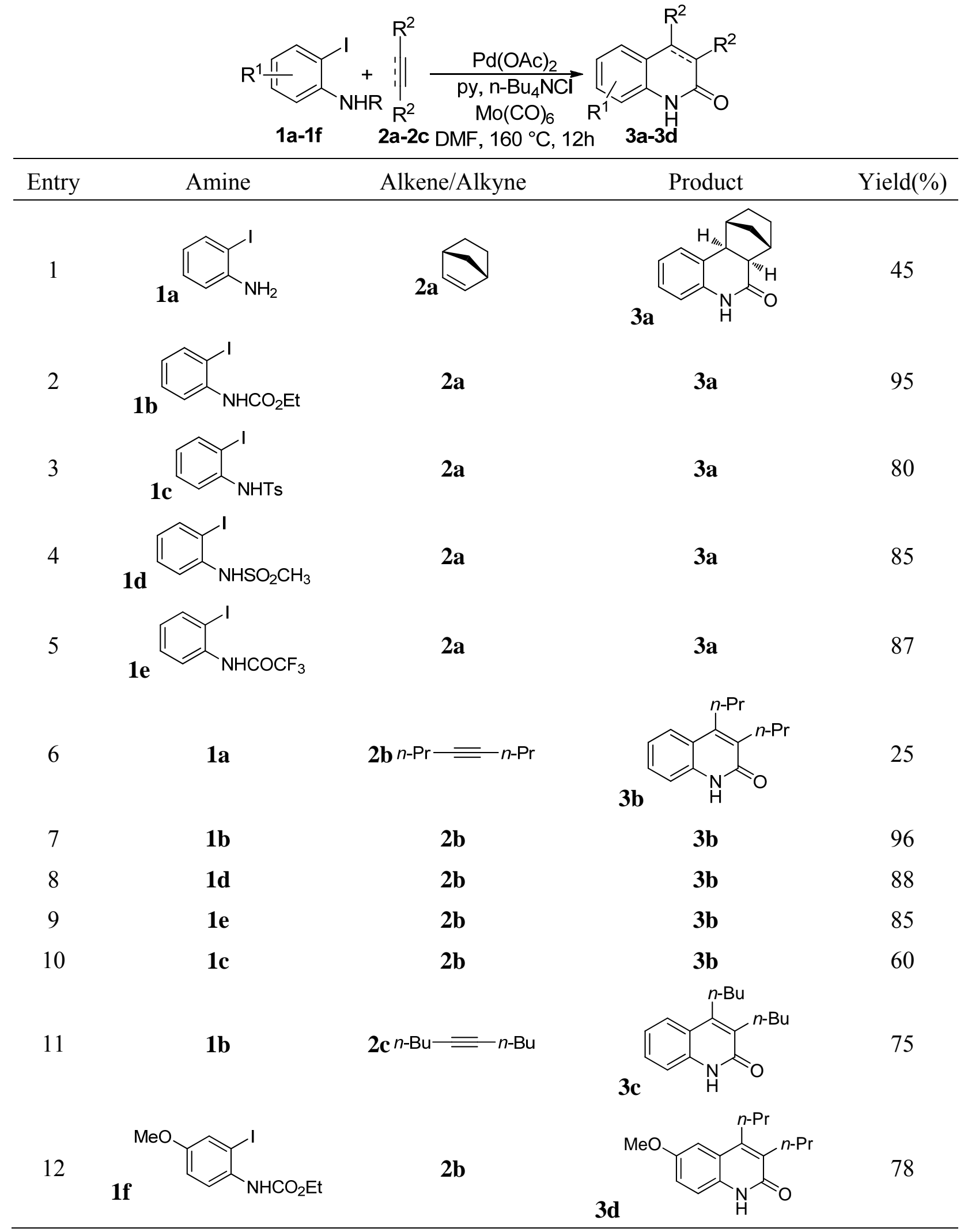


When aniline with tosyl protecting group was used in the reaction, a moderate yield of the desired product was obtained (entry 10). 5-Decyne also was employed in annulation reaction and afforded product 3c in high yield (entry 11). Next the reactivity of substituted iodoaniline was examined in the carbonylative annulation reaction of alkynes. Accordingly, o-iodoaniline $\mathbf{1 f}$ was reacted with 4-octyene and the desired quinolone 3d was obtained in $78 \%$ isolated yield (entry 12). Unfortunately, diphenylacetylene did not participate in this annulation reaction and only traces of the desired product were obtained. Homo-coupling and polymerization of diarylacetylene, could not be overcome during the liberation of carbon monoxide in the course of reaction.

\section{Conclusions}

We have developed a versatile and efficient route to 3,4-disubstituted 2-quinolones via palladium catalyzed carbonylative annulation of internal alkynes as well as norbornene under mild reaction conditions. The presented methodology employs readily available starting materials including iodoanilines, unsaturated compounds and $\mathrm{Mo}(\mathrm{CO})_{6}$ and establishes $\mathrm{CO}$ gas free conditions which seems to overcome the trouble of using gaseous carbon monoxide and looks suitable for highthroughput carbonylative reactions. Furthermore, the reaction is compatible with various Nprotected anilines and results in deprotected amines in the course of the reaction.

\section{Experimental Section}

General. All reagents and metal catalysts were commercially available and used as received. All carbonylative annulation reactions were carried out in an oil bath using Microwave Vials (2-5 $\mathrm{mL}$ ). IR spectra were recorded on a Shimadzu FT-IR 4300 spectrometer. IR is reported as characteristic bands $\left(\mathrm{cm}^{-1}\right)$ in their maximal intensity. ${ }^{1} \mathrm{H}$ and ${ }^{13} \mathrm{C}$ NMR spectra were recorded at room temperature on a Bruker $\mathrm{AC} 300 \mathrm{MHz}$ or $500 \mathrm{MHz}$ spectrometers using $\mathrm{CDCl}_{3}$ as the NMR solvent. Mass spectra of the products were obtained with a HP (Agilent Technologies) 5937 Mass Selective Detector. Elemental analyses were obtained using a Flash EA 1112 elemental analysis instrument.

General procedure for the Pd-catalyzed synthesis of 3,4-disubstituted 2-quinolones. A vial equipped with a stir bar was charged with 2-iodoaniline ( $0.2 \mathrm{mmol}, 1.0$ equiv), alkene or alkyne $(1.0 \mathrm{mmol})$, pyridine $(0.6 \mathrm{mmol}), n-\mathrm{Bu}_{4} \mathrm{NCl}(0.2 \mathrm{mmol}), \mathrm{Pd}(\mathrm{OAc})_{2}(10 \mathrm{~mol} \%)$ and $\mathrm{Mo}(\mathrm{CO})_{6}$ $(0.3 \mathrm{mmol})$. DMF $(2 \mathrm{~mL})$ was then added and the vial was capped. The resulting mixture was heated in an oil bath at $160{ }^{\circ} \mathrm{C}$ for $12 \mathrm{~h}$. After completion of the reaction, the reaction mixture was cooled to room temperature. The residue was treated with $2 \mathrm{~mL}$ of $1 \mathrm{M}$ ethanolic $\mathrm{NaOH}$ at room temperature for $30 \mathrm{~min}$. The resulting mixture was then extracted with DCM. Organic 
fractions were combined, washed with brine, dried over anhydrous $\mathrm{MgSO}_{4}$, and concentrated under reduced pressure. The residue was separated by column chromatography on silica gel.

6a,7,8,9,10,10a-Hexahydro-7,10-methanophenanthridin-6(5H)-one (3a). White solid, mp 161-163 ${ }^{\circ} \mathrm{C}$; IR $\left(v_{\max }, \mathrm{cm}^{-1}\right): 1665(\mathrm{C}=\mathrm{O}) .{ }^{1} \mathrm{H}$ NMR $\left(500 \mathrm{MHz}, \mathrm{CDCl}_{3}\right): \delta_{\mathrm{H}} 1.18(1 \mathrm{H}, \mathrm{d}, J 10.3$ $\mathrm{Hz}, \mathrm{CH}), 1.45$ (1H, d, J $10.3 \mathrm{~Hz}, \mathrm{CH}), 1.54(2 \mathrm{H}, \mathrm{m}, 2 \mathrm{CH}), 1.69(2 \mathrm{H}, \mathrm{m}, 2 \mathrm{CH}), 2.45$ (1H, d, J 2.7 $\mathrm{Hz}, \mathrm{CH}), 2.74(1 \mathrm{H}, \mathrm{d}, J 9.8 \mathrm{~Hz}, \mathrm{CH}), 2.88(1 \mathrm{H}, \mathrm{d}, J 3.1 \mathrm{~Hz}, \mathrm{CH}), 3.13(1 \mathrm{H}, \mathrm{d}, J 9.8 \mathrm{~Hz}, \mathrm{CH})$, $6.72(1 \mathrm{H}, \mathrm{d}, J 7.8 \mathrm{~Hz}, \mathrm{Ar}-\mathrm{H}), 6.98$ (1H, t, J 7.3 Hz, Ar-H), 7.09 (1H, t, J 7.4 Hz, Ar-H), 7.16 (1H, $\mathrm{d}, J 7.5 \mathrm{~Hz}, \mathrm{Ar}-\mathrm{H}), 9.12(1 \mathrm{H}, \mathrm{s}, \mathrm{NH}) .{ }^{13} \mathrm{C} \mathrm{NMR}\left(75 \mathrm{MHz}, \mathrm{CDCl}_{3}\right): \delta_{\mathrm{C}} 28.9,29.6,34.7,44.2,45.5$, 48.1, 48.6, 115.4, 123.1, 124.0, 127.1, 129.2, 136.0, $171.7(\mathrm{C}=\mathrm{O}) . \mathrm{MS}, \mathrm{m} / \mathrm{z}(\%)=213\left(\mathrm{M}^{\circ+}, 70\right)$, 196 (34), 146 (100), 76 (23). Anal. Calcd for $\mathrm{C}_{14} \mathrm{H}_{15} \mathrm{NO}$ (213.28): C, 78.84; H, 7.09; N, 6.57\%. Found: C, 79.11; H, 7.20; N, 6.71\%.

3,4-Dipropyl-2(1H)-quinolinone (3b): White solid, mp 188-190 ${ }^{\circ} \mathrm{C}$ (Ref. ${ }^{67} 159-160{ }^{\circ} \mathrm{C}$ ); IR $\left(v_{\max }, \mathrm{cm}^{-1}\right): 1651(\mathrm{C}=\mathrm{O}) .{ }^{1} \mathrm{H}$ NMR $\left(300 \mathrm{MHz}, \mathrm{CDCl}_{3}\right): \delta_{\mathrm{H}} 1.06-1.12\left(6 \mathrm{H}, \mathrm{m}, 2 \mathrm{CH}_{3}\right), 1.63-1.67$ (4H, m, $\left.2 \mathrm{CH}_{2}\right), 2.73-2.77\left(2 \mathrm{H}, \mathrm{m}, \mathrm{CH}_{2}\right), 2.86-2.90\left(2 \mathrm{H}, \mathrm{m}, \mathrm{CH}_{2}\right), 7.18-7.22(1 \mathrm{H}, \mathrm{m}, \mathrm{Ar}-\mathrm{H}), 7.40-$ $7.44(2 \mathrm{H}, \mathrm{m}, \mathrm{Ar}-\mathrm{H}), 7.68(1 \mathrm{H}, \mathrm{d}, J 8.1 \mathrm{~Hz}, \mathrm{Ar}-\mathrm{H}), 12.17(1 \mathrm{H}, \mathrm{s}, \mathrm{NH}) .{ }^{13} \mathrm{C} \mathrm{NMR}(75 \mathrm{MHz}$, $\left.\mathrm{CDCl}_{3}\right): \delta_{\mathrm{C}} 14.5,14.6,22.7,23.3,29.1,30.9,116.3,120.1,122.0,124.3,129.0,131.2,137.3$, 147.3, $164.0(\mathrm{C}=\mathrm{O}) . \mathrm{MS}, \mathrm{m} / \mathrm{z}(\%)=229\left(\mathrm{M}^{\bullet+}, 49\right), 214(62), 186(36), 129(46), 83$ (58), 71 (61), 57 (100), 43 (95). Anal. Calcd for $\mathrm{C}_{15} \mathrm{H}_{19} \mathrm{NO}$ (229.32): C, 78.56; H, 8.35; N, 6.11. Found: C, $78.80 ; \mathrm{H}, 8.47$; N, 6.27.

3,4-Dibutyl-2(1H)-quinolinone (3c): White solid, mp 133-134 ${ }^{\circ} \mathrm{C}\left(\operatorname{Ref}^{63} 134-135{ }^{\circ} \mathrm{C}\right)$; IR $\left(v_{\max }\right.$, $\left.\mathrm{cm}^{-1}\right) 1656(\mathrm{C}=\mathrm{O}) .{ }^{1} \mathrm{H}$ NMR $\left(300 \mathrm{MHz}, \mathrm{CDCl}_{3}\right): \delta_{\mathrm{H}} 0.98-1.04\left(6 \mathrm{H}, \mathrm{m}, 2 \mathrm{CH}_{3}\right), 1.49-1.68(8 \mathrm{H}, \mathrm{m}$, 4CH $\left.\mathrm{CH}_{2}\right), 2.79\left(2 \mathrm{H}, \mathrm{t}, J 7.2 \mathrm{~Hz}, \mathrm{CH}_{2}\right), 2.91\left(2 \mathrm{H}, \mathrm{t}, J 7.7 \mathrm{~Hz}, \mathrm{CH}_{2}\right), 7.22(1 \mathrm{H}, \mathrm{t}, J 7.1 \mathrm{~Hz}, \mathrm{Ar}-\mathrm{H})$, 7.40-7.46 (2H, m, Ar-H), $7.69(1 \mathrm{H}, \mathrm{d}, J 8.1 \mathrm{~Hz}, \mathrm{Ar}-\mathrm{H}), 12.3(1 \mathrm{H}, \mathrm{s}, \mathrm{NH}) .{ }^{13} \mathrm{C} \mathrm{NMR}(75 \mathrm{MHz}$, $\left.\mathrm{CDCl}_{3}\right): \delta_{\mathrm{C}} 14.0,14.2,23.2,23.4,26.8,28.7,31.7,32.2,116.4,120.2,122.1,124.3,129.0,131.4$, 137.4, 147.5, $164.1(\mathrm{C}=\mathrm{O})$. Anal. Calcd for $\mathrm{C}_{17} \mathrm{H}_{23} \mathrm{NO}$ (257.37): C, 79.33; H, 9.01; N, 5.44. Found: C, 79.60; H, 9.13; N, 5.58.

6-Methoxy-3,4-dipropyl-2(1H)-quinolinone (3d): Brown solid, mp 157-158 ${ }^{\circ} \mathrm{C}$ (Ref. ${ }^{67} 158$ $\left.161{ }^{\circ} \mathrm{C}\right)$; IR $\left(v_{\max }, \mathrm{cm}^{-1}\right) 1650(\mathrm{C}=\mathrm{O}) .{ }^{1} \mathrm{H} \mathrm{NMR}\left(300 \mathrm{MHz}, \mathrm{CDCl}_{3}\right): \delta_{\mathrm{H}} 1.09(3 \mathrm{H}, \mathrm{t}, J 7.3 \mathrm{~Hz}$, $\left.\mathrm{CH}_{3}\right), 1.11\left(3 \mathrm{H}, \mathrm{t}, J 7.3 \mathrm{~Hz}, \mathrm{CH}_{3}\right), 1.60-1.70\left(4 \mathrm{H}, \mathrm{m}, 2 \mathrm{CH}_{2}\right), 2.75-2.78\left(2 \mathrm{H}, \mathrm{m}, \mathrm{CH}_{2}\right), 2.84-2.88$ $\left(2 \mathrm{H}, \mathrm{m}, \mathrm{CH}_{2}\right), 3.88\left(3 \mathrm{H}, \mathrm{s}, \mathrm{OCH}_{3}\right), 7.08-7.12(2 \mathrm{H}, \mathrm{m}, \mathrm{Ar}-\mathrm{H}), 7.34-7.37(1 \mathrm{H}, \mathrm{m}, \mathrm{Ar}-\mathrm{H}), 12.1(1 \mathrm{H}$, $\mathrm{s}, \mathrm{NH}) .{ }^{13} \mathrm{C}$ NMR $\left(75 \mathrm{MHz}, \mathrm{CDCl}_{3}\right): \delta_{\mathrm{C}} 14.9,15.0,23.0,23.4,29.5,31.3,56.0,107.4,117.7$, 117.8, 121.1, 132.0, 132.3, 147.1, 155.1, $164.0(\mathrm{C}=\mathrm{O})$. Anal. Calcd for $\mathrm{C}_{16} \mathrm{H}_{21} \mathrm{NO}_{2}$ (259.34): $\mathrm{C}$, 74.10; H, 8.16; N, 5.40. Found: C, 74.38; H, 8.29; N, 5.57.

\section{Acknowledgements}

We thank the Research Council of the University of Tehran and Iran National Science Foundation (INSF) for financial support of this project. 


\section{References}

1. Wu, X. F.; Neumann, H.; Beller, M. Chem. Rev. 2013, 113, 1. http://dx.doi.org/10.1021/cr300100s

2. Liu, Q.; Zhang, H.; Lei, A. Angew. Chem. Int. Ed. 2011, 50, 10788. http://dx.doi.org/10.1002/anie.201100763

3. Wu, X. F.; Neumann, H.; Beller, M. Chem. Soc. Rev. 2011, 40, 4986. http://dx.doi.org/10.1039/c1cs15109f

4. Grigg, R.; Mutton, S. P. Tetrahedron 2010, 66, 5515. http://dx.doi.org/10.1016/j.tet.2010.03.090

5. Brennführer, A.; Neumann, H.; Beller, M. Angew. Chem. Int. Ed. 2009, 48, 4114. http://dx.doi.org/10.1002/anie.200900013

6. Barnard, C. F. J. Organometallics 2008, 27, 5402. http://dx.doi.org/10.1021/om800549q

7. Schoenberg, A.; Heck, R. F. J. Org. Chem. 1974, 39, 3327. http://dx.doi.org/10.1021/jo00937a004

8. Schoenberg, A.; Bartoletti, I.; Heck, R. F. J. Org. Chem. 1974, 39, 3318. http://dx.doi.org/10.1021/jo00937a003

9. Wu, X. F.; Neumann, H.; Beller, M. Chem. Eur. J. 2012, 18, 12599. http://dx.doi.org/10.1002/chem.201202142

10. Wu, X. F.; Schranck, J.; Neumann, H.; Beller, M. Chem. Eur. J. 2011, 17, 12246. http://dx.doi.org/10.1002/chem.201102254

11. Giri, R.; Lam, J. K.; Yu, J. Q. J. Am. Chem. Soc. 2010, 132, 686. http://dx.doi.org/10.1021/ja9077705

12. Salvadori, J.; Balducci, E.; Zaza, S.; Petricci, E.; Taddei, M. J. Org. Chem. 2010, 75, 1841. http://dx.doi.org/10.1021/jo9021315

13. Merkul, E.; Oeser, T.; Müller, T. J. J. Chem. Eur. J. 2009, 15, 5006. http://dx.doi.org/10.1002/chem.200900119

14. Willy, B.; Müller, T. J. J. Curr. Org. Chem. 2009, 13, 1777. http://dx.doi.org/10.2174/138527209789630479

15. Houlden, C. E.; Hutchby, M.; Bailey, C. D.; Ford, J. G.; Tyler, S. N. g.; Gagne, M. R.; Loyd-Jones, G. C.; Booker-Milburn, K. I. Angew. Chem. Int. Ed. 2009, 48, 1830. http://dx.doi.org/10.1002/anie.200805842

16. Willy, B.; Müller, T. J. J. Eur. J. Org. Chem. 2008, 24, 4157. http://dx.doi.org/10.1002/ejoc.200800444

17. Wannberg, J.; Larhed, M. In: Modern Carbonylation Methods; Kollar, L. Ed.; Wiley-VCH: Weinheim, 2008; p 93. http://dx.doi.org/10.1002/9783527621545.ch4

18. Wu, X.-F.; Sharif, M.; Shoaib, K.; Neumann, H.; Pews-Davtyan, A.; Langer, P.; Beller, M. Chem. Eur. J. 2013, 19, 6230. 
http://dx.doi.org/10.1002/chem.201300537

19. Borhade, S. R.; Sandström, A.; Arvidsson, P. I. Org. Lett. 2013, 15, 1056.

http://dx.doi.org/10.1021/o1400049m

20. Iranpoor, N.; Firouzabadi, H.; Motevalli, S.; Talebi, M. Tetrahedron 2013, 69, 418. http://dx.doi.org/10.1016/j.tet.2012.10.002

21. Odell, L. R.; Russo, F.; Larhed, M. Synlett 2012, 685. http://dx.doi.org/10.1055/s-0031-1290350

22. Nordeman, P.; Odell, L. R.; Larhed, M. J. Org. Chem. 2012, 77, 11393. http://dx.doi.org/10.1021/jo302322w

23. Pyo, A.; Park, A.; Jung, H. M.; Lee, S. Synthesis 2012, 44, 2885. http://dx.doi.org/10.1055/s-0032-1316760

24. Begouin, A.; Queiroz, M.-J. R. P. Eur. J. Org. Chem. 2009, 2820. http://dx.doi.org/10.1002/ejoc.200900167

25. Wieckowska, A.; Fransson, R.; Odell, L. R.; Larhed, M. J. Org. Chem. 2011, 76, 978. http://dx.doi.org/10.1021/jo102151u

26. Sawant, D. N.; Wagh, Y. S.; Bhatte, K. D.; Bhanage, B. M. J. Org. Chem. 2011, 76, 5489. http://dx.doi.org/10.1021/jo200754v

27. Sävmarker, J.; Lindh, J.; Nilsson, P. Tetrahedron Lett. 2010, 51, 6886. http://dx.doi.org/10.1016/j.tetlet.2010.10.115

28. Ren, W.; Yamane, M. J. Org. Chem. 2010, 75, 8410. http://dx.doi.org/10.1021/jo101611g

29. Lindh, J.; Fardost, A.; Almeida, M.; Nilsson, P. Tetrahedron Lett. 2010, 51, 2470. http://dx.doi.org/10.1016/j.tetlet.2010.02.165

30. Liptrot, D.; Alcaraz, L.; Roberts, B. Adv. Synth. Catal. 2010, 352, 2183. http://dx.doi.org/10.1002/adsc.201000395

31. Lagerlund, O.; Mantel, M. L. H.; Larhed, M. Tetrahedron 2009, 65, 7646. http://dx.doi.org/10.1016/j.tet.2009.06.101

32. Odell, L. R.; Sävmarker, J.; Larhed, M. Tetrahedron Lett. 2008, 49, 6115. http://dx.doi.org/10.1016/j.tetlet.2008.08.014

33. Jafarpour, F.; Rashidi-Ranjbar, P.; Kashani, A. O. Eur. J.Org. Chem. 2011, 2128.

34. Sabbah, D. A.; Simms, N. A.; Wang, W.; Dong, Y. X.; Ezell, E. L.; Brattain, M. G.; Vennerstrom, J. L.; Zhong, H. A. Bioorg. Med. Chem. 2012, 20, 7175.

http://dx.doi.org/10.1016/j.bmc.2012.09.059

35. Claassen, G.; Brin, E.; Crogan-Grundy, C.; Vaillancourt, M. T.; Zhang, H. Z.; Cai, S. X.; Drewe, J.; Tseng, B.; Kasibhatla, S. Cancer Lett. 2009, 274, 243. http://dx.doi.org/10.1016/j.canlet.2008.09.032

36. Zhao, S.; Shen, Y.; van Oeveren, A.; Marschke, K. B.; Zhi, L. Bioorg. Med. Chem. Lett. 2008, 18, 3431.

http://dx.doi.org/10.1016/j.bmcl.2008.03.085 
37. Detsi, A.; Bouloumpasi, D.; Proussis, K. C.; Koufaki, M.; Athanasellis, G.; Melagraki, G.; Afantitis, A.; Igglessi-Markopoulou, O.; Kontogiorgis, C.; Hadjipavlou-Litina, D. J. J. Med. Chem. 2007, 50, 2450.

38. Marull, M.; Lefebvre, O.; Schlosser, M. Eur. J. Org. Chem. 2004, 54. http://dx.doi.org/10.1002/ejoc.200300531

39. Reddy, M. S.; Thirupathi, N.; Babu, M. H. Eur. J. Org. Chem. 2012, 5803. http://dx.doi.org/10.1002/ejoc.201200782

40. Gao, W.-T.; Hou, W.-D.; Zheng, M.-R.; Tang, L.-J. Synth. Commun. 2010, 40, 732. http://dx.doi.org/10.1080/00397910903013713

41. Dominguez-Fernandez, F.; Lopez-Sanz, J.; Perez-Mayoral, E.; Bek, D.; Martin-Aranda, R. M.; Lopez-Peinado, A. J.; Cejka, J. Chem.Cat.Chem. 2009, 1, 241.

42. Yasui, Y.; Kakinokihara, I.; Takeda, H.; Takemoto, Y. Synthesis 2009, 23, 3989.

43. Huang, C.-C.; Chang, N.-C. Org. Lett. 2008, 10, 673. http://dx.doi.org/10.1021/o17030312

44. Ryabukhin, D. S.; Vasil'ev, A. V. Russ. J. Org. Chem. 2008, 44, 1849. http://dx.doi.org/10.1134/S1070428008120257

45. Angibaud, P. R.; Venet, M. G.; Filliers, W.; Broeckx, R.; Ligny, Y. A.; Muller, P.; Poncelet, V. S.; End, D. W. Eur. J. Org. Chem. 2004, 479.

http://dx.doi.org/10.1002/ejoc.200300538

46. Park, K. K.; Lee, J. J. Tetrahedron 2004, 60, 2993. http://dx.doi.org/10.1016/j.tet.2004.02.001

47. Wang, J.; Discordia, R. P.; Crispino, G. A.; Li, J.; Grosso, J. A.; Polniaszek, R.; Truc, V. C. Tetrahedron Lett. 2003, 44, 4271. http://dx.doi.org/10.1016/S0040-4039(03)00889-X

48. Ferguson, J.; Zeng, F.; Alwis, N.; Alper, H. Org. Lett. 2013, 15, 1998. http://dx.doi.org/10.1021/o14006739

49. Inamoto, K.; Kawasaki, J.; Hiroya, K.; Kondo, Y.; Doi, T. Chem. Commun. 2012, 48, 4332. http://dx.doi.org/10.1039/c2cc30600j

50. Shibuya, T.; Shibata, Y.; Noguchi, K.; Tanaka, K. Angew. Chem., Int. Ed. 2012, 50, 3963. http://dx.doi.org/10.1002/anie.201100152

51. Fu, L.; Huang, X.; Wang, D.; Zhao, P.; Ding, K. Synthesis 2011, 1547.

52. Kato, H.; Ishigame, T.; Oshima, N.; Hoshiya, N.; Shimawaki, K.; Arisawa, M.; Shuto, S. Adv. Synth. Catal. 2011, 353, 2676.

http://dx.doi.org/10.1002/adsc.201100303

53. Tang, B.-X.; Song, R.-J.; Wu, C.-Y.; Wang, Z.-Q.; Liu, Y.; Huang, X.-C.; Xiea, Y.-X.; Li, J.-H. Chem. Sci. 2011, 2, 2131. http://dx.doi.org/10.1039/c1sc00423a

54. Borhade, S. R.; Waghmode, S. B. Can. J. Chem. 2011, 89, 1355. http://dx.doi.org/10.1139/v11-103

55. Harishkumar, H. N.; Hulikal, V. K.; Mahadevan, K. M. Synth. Commun. 2010, 40, 3281 
http://dx.doi.org/10.1080/00397910903399690

56. Tang, D.-J.; Tang, B.-X.; Li, J.-H. J. Org. Chem. 2009, 74, 6749.

http://dx.doi.org/10.1021/jo901314t

57. Kobayashi, Y.; Harayama, T. Org. Lett. 2009, 11, 1603.

http://dx.doi.org/10.1021/o1900255g

58. Tadd, A. C.; Matsuno, A.; Fielding, M. R.; Willis, M. C. Org. Lett. 2009, 11, 583. http://dx.doi.org/10.1021/01802624e

59. Wasa, M.; Yu, J.-Q. J. Am. Chem. Soc. 2008, 130, 14058.

http://dx.doi.org/10.1021/ja807129e

60. Wang, Z.; Fan, R.; Wu, J. Adv. Synth. Catal. 2007, 349, 1943.

http://dx.doi.org/10.1002/adsc.200700038

61. Nakai, K.; Kurahashi, T.; Matsubara, S. Org. Lett. 2013, 15, 856.

http://dx.doi.org/10.1021/o1303546p

62. Nakai, K.; Kurahashi, T.; Matsubara, S. J. Am. Chem. Soc. 2011, 133, 11066.

http://dx.doi.org/10.1021/ja203829j

63. Iwai, T.; Fujihara, T.; Terao, J.; Tsuji, Y. J. Am. Chem. Soc. 2010, 132, 9602.

http://dx.doi.org/10.1021/ja104153k

64. Minville, J.; Poulin, J.; Dufresne, C.; Sturino, C. F. Tetrahedron Lett. 2008, 49, 3677. http://dx.doi.org/10.1016/j.tetlet.2008.03.144

65. Battistuzzi, G.; Bernini, R.; Cacchi, S.; De Salve, I.; Fabrizi, G. Adv. Synth. Catal. 2007, 349, 297.

http://dx.doi.org/10.1002/adsc.200600342

66. Inamoto, K.; Saito, T.; Hiroya, K.; Doi, T. J. Org. Chem. 2010, 75, 3900.

http://dx.doi.org/10.1021/jo100557s

67. Kadnikov, D. V.; Larock, R. C. J. Org. Chem. 2004, 69, 6772.

http://dx.doi.org/10.1021/jo049149+

68. Kadnikov, D. V.; Larock, R. C. J. Organomet. Chem. 2003, 687, 425.

http://dx.doi.org/10.1016/S0022-328X(03)00786-1

69. Chen, J.-R.; Liao, J.; Xiao, W.-J. Can. J. Chem. 2010, 88, 331. 